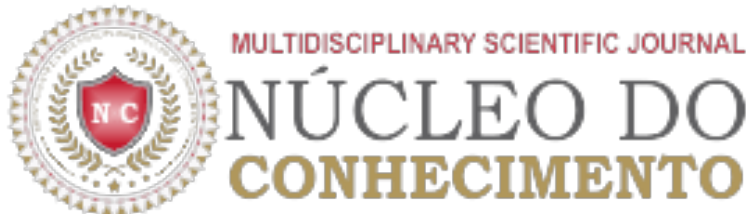

\section{Estudo Sobre o Estadiamento da Insuficiência Renal}

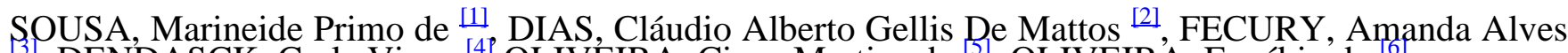
3], DENDASCK, Carla Viana ${ }^{[4]}$,OLIVEIRA, Ciane Martins de ${ }^{1]}$, OLIVEIR'A, Euzébio de ${ }^{[6]}$

SOUSA, Marineide Primo de. Et.al. Estudo Sobre o Estadiamento da Insuficiência Renal. Revista Científica Multidisciplinar Núcleo do Conhecimento. Ed. 11, Ano 02, Vol. 04. pp. 53-67, Novembro de 2017. ISSN:2448-0959, Link de acesso: https://www.nucleodoconhecimento.com.br/saude/insuficienciarenal, DOI: 10.32749/nucleodoconhecimento.com.br/saude/insuficiencia-renal

\section{RESUMO}

O rim é o órgão responsável pelo controle do equilíbrio hidro-eletrolítico e ácido-básico de nosso organismo. A insuficiência renal aguda pode ser causada por hipofluxo renal, lesão do próprio parênquima renal e obstrução do sistema uro-excretor. A insuficiência renal crônica é o estado de disfunção renal persistente, irreversível. Objetivo: aprofundar as funções básicas dos rins, os sintomas e as fases do estadiamento da insuficiência real desde o seu diagnóstico até o estágio da falência renal crônica, a fim aprender orientar para a prevenção da doença. Metodologia: pesquisa bibliográfica. Resultados: O novo sistema de estadiamento da doença renal crônica da National Kidney Foundation considera cinco estágios, desde a lesão renal crônica em insuficiência até a falência renal crônica. Quando se trata do estadiamento da insuficiência renal não se pode esquecer de realizar o diagnóstico precoce da doença, o encaminhamento imediato para tratamento nefrológico e a implementação de medidas para preservar a função renal. Conclusão: É recomendável a pacientes com mais de 40 anos fazerem anualmente uma consulta com um médico nefrologista, exames de dosagem da creatinina no sangue e o exame de urina.

Palavras-Chave: Insuficiência Renal, Estadiamento, Terapias para Tratamento.

\section{INTRODUÇÃO}

A palavra Nefrologia é originada na língua grega (nephros), significa "rim", e representa uma especialidade médica muito importante. Nefrologia é uma especialidade médica dedicada ao diagnóstico e tratamento clínico das doenças do sistema urinário, principalmente relacionadas ao rim (SOCIEDADE BRASILEIRA DE NEFROLOGIA, 2013). O médico especializado nas doenças do sistema urinário chama-se nefrologista. O tempo para formar um nefrologista é 10 anos de estudo ao todo. A Nefrologia 
no Brasil tem realizado muitas conquistas, desde o início nos anos de 1960, quando seu foco foi a Terapia Renal Substitutiva (TRS) - diálise e transplante renal. Mas quais são os fatores de risco críticos para perda da função renal? Quais são as orientações e estratégias para o tratamento e cuidados para a população?

Segundo o Ministério da Saúde, as doenças circulatórias são responsáveis por impacto expressivo na mortalidade da população brasileira, correspondendo a 32\% dos óbitos em 2002, o equivalente a 267.496 mortes (BRASIL, 2006). Mas quais são os fatores, principais grupos de riscos e estadiamento da Insuficiência Renal Crônica (IRC)?

Em Nefrologia o termo estadiamento é muito usado, sendo compreendido como uma maneira de avaliar a extensão da doença em relação ao órgão de origem, e, por conseguinte, avaliar qual o grau de comprometimento do órgão no qual o tumor teve início e avaliar se a doença se espalhou além do local de origem. Dessa maneira, é imprescindível o profissional em Nefrologia considerar a história clínica (queixas, sintomas, antecedentes pessoais e familiares) e o exame físico do paciente. Júnior (2004) aponta que para efeitos clínicos, epidemiológicos, didáticos e conceituais, a IRC é dividida em seis estágios funcionais, de acordo com o grau de função renal do paciente, indo da fase renal normal sem lesão renal até a terminal.

Assim, o presente trabalho teve como objetivo descrever as funções básicas dos rins, os sintomas e as fases do estadiamento da insuficiência real desde o seu diagnóstico até o estágio da falência renal crônica, a fim aprender orientar para a prevenção da doença.

\section{FUNDAMENTAÇÃO TEÓRICA}

\subsection{DESCRIÇÃO ANATÔMICA DOS RINS}

Anatomicamente, o rim é suprido por uma única artéria, denominada renal principal, com posição e trajeto relativamente constantes até formar o hilo. A origem encontra-se na aorta abdominal entre os níveis de L1 e L2 que constituem a coluna lateral dos segmentos medulares torácicos e lombares altos (OZKAN et al., 2006). Há diferenças no tamanho entre a artéria renal direita e a esquerda, em adultos, enquanto a direita apresenta trajeto de cerca de $5 \mathrm{~cm}$, a esquerda, tem $7 \mathrm{~cm}$. As duas são divididas próximas ao hilo em dois, três ou quatro ramos terminais. São originadas uma ou mais artérias suprarrenais de cada artéria renal, além de um ramo para o ureter e de diversos ramos para o tecido adjacente e retroperitônio (TESTUT e LATARJET, 1975 apud PALMIERI et al., 2011).

De acordo com Satyapal et al. (2001), variações anatômicas dessas artérias não interferem na função renal e devem ser diferenciadas de anomalias ou malformações vasculares, que provocam distúrbios funcionais renais e sistêmicos. Como afirmam Palmieri et al. (2011), houve vezes em que as variações nas artérias receberam termos como acessórias, aberrantes, anômalas, supranumerárias e suplementares. Para Sampaio e Passos (1992), pode-se denominar essas artérias de múltiplas, pois devido não terem anastomoses entre si constituem-se vasos segmentares para os rins.

Conforme Moon et al. (1998), geralmente, as artérias que se dirigem aos pólos renais são menos calibrosas que as artérias renais hilares, provenientes das artérias renais principais.

Para Bordei et al. (2004), deve-se realizar uma avaliação tanto da presença de múltiplas artérias renais, 
assim como o padrão de suas divisões pré-hilares, por causa da importância em relação à irrigação renal e por influenciar nos planos de dissecção e acesso ao hilo renal.

Para Motta (2011), o néfron é a unidade organizacional básica do rim e consiste num leito capilar especializado, o glomérulo, envolvido pelo epitélio urinário (cápsula de Bowman) e conectado a uma sucessão de segmentos epiteliais especializados, os túbulos. Cada rim humano contém cerca de 1,2 milhão de néfrons. O néfron é responsável por dois processos em série: ultrafiltração glomerular e a reabsorção/secreção tubular.

\subsection{FUNÇÕES RENAIS}

Segundo a Sociedade Brasileira de Nefrologia (2013), os rins, mesmo pequenos, com tamanho aproximado de $10 \mathrm{~cm}$, realizam o importante trabalho de fazer o balanço sadio da química interna do corpo. Para uma sobrevivência sadia, esses órgãos devem funcionar normalmente, desempenhando quatro funções: eliminação de toxinas do sangue por um sistema de filtração, regulação da formação do sangue e da produção dos glóbulos vermelhos, regulação da pressão sanguínea e controle do delicado balanço químico e de líquidos do corpo.

\subsubsection{A FUNÇÃO DE FILTRO: EXCREÇÃO DE TOXINAS}

O rim tem a função de eliminar do organismo a maior parte das substâncias tóxicas derivadas do nosso metabolismo. As principais toxinas, formadas diariamente, são derivadas do metabolismo proteico. Essas substâncias contêm nitrogênio em suas moléculas, pois se originam da quebra dos aminoácidos e são chamadas, corriqueiramente, de escórias nitrogenadas ou compostos azotêmicos. A ureia, quando em concentrações muito altas (>380 mg/dL) também apresenta efeitos tóxicos, como náuseas, vômitos, anorexia e sangramento (ENGEL et al., 2005).

O rim executa a sua função excretória através da filtração glomerular. A Taxa de Filtração Glomerular (TFG) é o parâmetro que quantifica esta função. O valor normal está entre $80-140 \mathrm{~mL} / \mathrm{min}$, o que equivale a aproximadamente 120 litros de plasma filtrados por dia. Destes, cerca de 118 litros retornam para o plasma através de reabsorção tubular; sendo os 2 litros restantes eliminados como urina (que contém todas as toxinas que precisam ser eliminadas do organismo) (ENGEL et al., 2005).

A filtração glomerular é a melhor medida do funcionamento renal em indivíduos normais ou pacientes com doença renal (KIDNEY DISEASE OUTCOME QUALITY INITIATIVE, K/DOQI, 2002). Para Bastos et al. (2010), quando a TFG atinge valores muito baixos, inferiores a $15 \mathrm{~mL} / \mathrm{min} / 1,73 \mathrm{~mm}^{3}$, estabelece-se o que denomina-se Falência Funcional Renal (FFR), ou seja, o estágio mais avançado do contínuo de perda funcional progressiva observado na DRC.

De maneira muito parecida ao trabalho dos filtros, os rins trabalham para conservar o corpo livre de toxinas. O sangue entra nos rins através da artéria renal. Uma vez que o sangue chega aos rins, as toxinas são filtradas para a urina. O sangue limpo volta ao coração por uma veia renal (SOCIEDADE BRASILEIRA DE NEFROLOGIA, 2013).

\subsubsection{PRODUÇÃO DE GLÓBULOS VERMELHOS E FORMAÇÃO DE OSSOS}


Segundo a Sociedade Brasileira de Nefrologia (2013), para formação dos ossos e a produção de glóbulos vermelhos, é necessário que os rins funcionem normalmente. Os rins regularizam as concentrações de cálcio e fósforo no sangue, além de produzirem uma forma ativa da Vitamina D. Também liberam eritropoetina, que auxilia na maturação dos glóbulos vermelhos no sangue e da medula óssea. Ratifica Motta (2011) é papel dos rins sintetizar eritropoietina, renina, prostaglandinas e 1,25-diidroxicolecalciferol (forma ativa da vitamina D).

\subsubsection{REGULAÇÃO DE PRESSÃO SANGUÍNEA}

Outra função importante dos rins é regular a pressão sanguínea. De acordo com a Sociedade Brasileira de Nefrologia (2013), faz-se necessário o controle das concentrações de sódio e a quantidade de líquido no corpo. Cabe aos rins secretarem uma substância que se chama renina. A renina estimula a produção de um hormônio que eleva a pressão sanguínea. Quando os rins não funcionam bem se produz renina em excesso e isto pode resultar em hipertensão. A hipertensão prolongada danifica os vasos sanguíneos, causando assim falha renal.

\subsubsection{CONTROLE DO BALANÇO QUÍMICO E DE LÍQUIDO DO CORPO}

Conforma a Sociedade Brasileira de Nefrologia (2013), quando os rins não funcionam apropriadamente, as toxinas se acumulam no sangue. Isto resulta em uma condição muito séria conhecida como uremia. Os sintomas da uremia incluem: náuseas, debilidade, fadiga, desorientação, dispneia e edema nos braços e pernas. Há toxinas que se acumulam no sangue e que podem ser usadas para avaliar a gravidade do problema. As principais substâncias comumente usadas para este propósito se chamam ureia e creatinina. A enfermidade dos rins está associada frequentemente com níveis elevados de ureia e de creatinina. Para Motta (2011), cabe ao rim regular o equilíbrio eletrolítico no líquido intersticial, controlando, simultaneamente, o movimento e a perda de água ao nível celular em colaboração com a pele e os pulmões.

\subsection{MEDIDA DA FUNÇÃO RENAL}

A única maneira de se medir a função excretória renal é pela quantificação da filtração glomerular. A síndrome urêmica aguda pode ocorrer com uma TGF menor que 15-30 mL/min (menos de 15-30\% da função renal), o que corresponde a uma concentração plasmática de ureia e creatinina acima de 120 $\mathrm{mg} / \mathrm{dL}$ e 4,0 mg/dL, respectivamente, em um adulto de 40 anos, pesando $70 \mathrm{~kg}$ (ENGEL et al., 2005).

Segundo Nunes (2007), os métodos mais comumente utilizados para estimar a TFG são concentração da creatinina sérica, Depuração da Creatinina Endógena (DCE), ou estimativa da TFG ou DCE por equações baseadas na creatinina sérica.

Na prática médica, pode-se quantificar a função renal utilizando-se os parâmetros relativos à função excretória. São eles: (1) ureia plasmática; (2) creatinina plasmática; (3) clearance de creatinina.

\subsubsection{UREIA PLASMÁTICA}

A ureia é formada no fígado a partir de moléculas de amônia $\left(\mathrm{NH}_{3}\right)$, produzida em grande quantidade pelo metabolismo proteico e pelas bactérias da flora intestinal. Enquanto a amônia é extremamente tóxica, 
sendo uma das substâncias causadoras do coma hepático, a toxicidade da ureia é controversa. Apenas em concentrações muito elevadas (>380 mg/dL) pode produzir efeitos adversos, geralmente relacionados ao trato gastro-intestinal e à hemostasia. No entanto, como a ureia é eliminada quase que exclusivamente pelo rim e é de fácil dosagem plasmática, pode-se utilizar a medida de seus níveis para avaliar a função excretória renal. De uma forma geral, os níveis de ureia se elevam para níveis acima dos valores de referência quando a TFG está menor que $50 \mathrm{~mL} / \mathrm{min}$. O valor normal da ureia é 20-40 mg/dL (ENGEL et al., 2005).

Entretanto, a ureia não é o melhor 'termômetro' para se medir a função renal. Uma parte da ureia filtrada pelo glomérulo é reabsorvida. Portanto, condições que aumentam a reabsorção tubular, como a hipovolemia, podem aumentar os níveis séricos de ureia, sem haver importante queda da função renal. Além disso, a grande produção de amônia após uma hemorragia digestiva, quando a flora intestinal cataboliza intensamente a hemoglobina liberada, leva a um aumento da produção hepática da ureia, elevando seus níveis (ENGEL et al., 2005).

Conforme Motta (2011), a ureia constitui 45\% do nitrogênio não proteico no sangue. Após a síntese hepática, a ureia é transportada pelo plasma até os rins, onde é filtrada pelos glomérulos. A ureia é excretada na urina, embora $40-70 \%$ seja reabsorvida por difusão passiva pelos túbulos. Um quarto da ureia é metabolizada no intestino para formar amônia e $\mathrm{CO}_{2}$, pela ação da flora bacteriana normal. Esta amônia é reabsorvida e levada ao fígado onde é reconvertida em ureia. O nível de ureia no plasma é afetado pela função renal, conteúdo proteico da dieta e teor do catabolismo proteico, estado de hidratação do paciente e presença de sangramento intestinal. Apesar destas limitações, entretanto, o nível de ureia ainda serve como um índice preditivo da insuficiência renal sintomática e no estabelecimento de diagnóstico na distinção entre várias causas de insuficiência renal.

\subsubsection{CREATINA PLASMÁTICA}

É uma substância não-tóxica produzida pelo tecido muscular, derivada da creatina, molécula armazenadora de energia. Tem grandes vantagens para ser utilizada como medida da função excretória renal: a) sua produção diária é relativamente constante; b) ao contrário do que ocorre com a ureia, não é reabsorvida pelo túbulo (ENGEL et al, 2005).

Para Stevens e Levey (2005), creatinina é um derivado de aminoácido com 113 dáltons, oriunda do metabolismo muscular e da ingestão de carne. É gerada no músculo a partir de uma reação não enzimática da creatina e fosfocreatina. A sua produção e liberação pelo músculo são praticamente constantes.

Os níveis normais de creatinina plasmática dependem da massa muscular do indivíduo. Em um indivíduo musculoso, uma creatinina plasmática de $1,3 \mathrm{mg} / \mathrm{dL}$ pode ser normal, enquanto que que em um desnutrido, a creatinina plasmática deve estar abaixo de $0,8 \mathrm{mg} / \mathrm{dL}$. O valor normal da creatinina é: Homens $<1,4 \mathrm{mg} / \mathrm{dL}$ e Mulheres $<1,2 \mathrm{mg} / \mathrm{dL}$ (ENGEL et al, 2005).

\subsubsection{CLEARANCE DE CREATINA}

Outro conceito importante é clearance, do inglês, significa depuração. Por definição, é o volume de plasma que fica livre da substância a ser eliminada a cada minuto. Por exemplo, o clearance da ureia é de 50-70 mL/min. Como uma parte da ureia é absorvida e retorna ao plasma, o seu clearance é menor que a 
TFG. A creatinina não é reabsorvida, tudo o que é filtrado no glomérulo e excretado na urina e, portanto, depurado do plasma. Pode-se dizer que o Clearance de Creatinina (CICr) é uma razoável estimativa da TFG apesar de superestimá-lo (costuma ser $15 \%$ maior que a TFG). O valor normal do $\mathrm{ClCr}$ é de 80-150 $\mathrm{mL} / \mathrm{min}$ (ENGEL et al., 2005).

De acordo com Godoy e Silva (2006) o Clearance de Creatinina (CLCR) continua sendo um dos marcadores mais usados na avaliação da função renal.

\subsection{UNSUFIÊNCIA RENAL AGUDA}

Ferraz e Deus (2009) conceituam a Insuficiência Renal Aguda (IRA) como sendo a perda súbita da capacidade do rim em manter sua atividade endócrina e exócrina, bem como a filtração glomerular e eliminação dos compostos descartados por esta filtração, produção e concentração urinária, manutenção da homeostase e equilíbrio ácido básico.

A IRA pode ser causada por três mecanismos básicos: (1) hipofluxo renal (azotemia pré-renal); (2) lesão do próprio parênquima renal (azotemia renal intrínseca) e, (3) obstrução do sistema uro-excretor (azotemia pós-renal).

A IRA é frequentemente observada em pacientes internados e sua prevalência está aumentando, principalmente na população idosa, com múltiplas comorbidades e em pacientes com doenças consideradas graves, como neoplasia, entre outras (NOLAN; ANDERSON, 2005).

\subsubsection{AZOTEMIA PRÉ-RENAL}

A azotemia pré-renal ou insuficiência pré-renal é a elevação das escórias nitrogenadas causada diretamente pela redução do fluxo sanguíneo renal. É o tipo mais comum de IRA (55-60\% dos casos). Caracteriza-se clinicamente pela reversibilidade, uma vez restaurado o fluxo renal. As principais causas são: (a) hipovolemia; (b) estados de choque; (c) insuficiência cardíaca; (d) cirrose hepática com ascite (ENGEL et al., 2005). As escórias nitrogenadas tais como: os compostos guanidínicos derivados da arginina, as aminas alifáticas ou aromáticas (derivadas do triptofano), os fenóis, os indóis, entre outros, causam as síndromes urêmicas (ETZEL, 2004).

Os vasos renais possuem um mecanismo de proteção contra alterações deletérias do fluxo renal e da TFG, o que denomina-se auto-regulação do fluxo renal e da filtração glomerular. Quando a Pressão Arterial Média (PAM) cai, as arteríolas aferentes dilatam, reduzindo a resistência vascular do rim, evitando o hipofluxo renal. Em condições normais, o fluxo sanguíneo renal é preservado até uma PAM de 80 mmHg. Caso esta pressão caia para abaixo desses valores, a auto-regulação não é mais capaz de evitar o hipofluxo, pois as arteríolas já estão no seu máximo de vasodilatação. Neste ponto, instala-se a azotemia pré-renal. É importante ressaltar que indivíduos com um desajuste da auto-regulação renal, podem desenvolver azotemia pré-renal mesmo com a PAM um pouco acima de $80 \mathrm{mmHg}$. É o caso de alguns idosos, hipertensos crônicos e diabéticos de longa data (ENGEL et al., 2005).

Para Nolan e Anderson (1998), a azotemia pré-renal constitui a causa isolada mais comum de IRA, responsável por 30 a $60 \%$ de todos os casos. A IRA pré-renal ocorre a partir da falha da perfusão glomerular, em decorrência de uma redução absoluta do volume de líquido extracelular ou de uma 
redução do volume circulante mesmo com a manutenção da normalidade do volume de líquido extracelular total, que podem ocorrer em condições como insuficiência ventricular esquerda, cirrose avançada e sepse. Na azotemia pré-renal o paciente está oligúrico, podendo apresentar hipotensão arterial e desidratação.

\subsection{AZOTEMIA PÓS-RENAL}

A azotemia pós-renal ou insuficiência renal é uma disfunção renal causada por obstrução aguda do sistema uro-excretor. É responsável por apenas 5\% dos casos de IRA, embora, no subgrupo de idosos, esta percentagem torne-se maior devido a maior prevalência da doença prostática (ENGEL et al., 2005).

Segundo Magro e Vattimo (2007) ao fazerem uma avaliação da função renal, creatinina e outros biomarcadores, apontaram que elevações nos níveis séricos da creatinina são atualmente os sinais mais indicativos de comprometimento da função renal. Apesar de representar a principal estratégia de identificação dessa síndrome, a creatinina é considerada um teste específico, entretanto, tardio, pouco sensível e impreciso. Ela se altera apenas quando já existe perda de aproximadamente $50 \%$ da função renal.

\subsection{UINSUFICIÊNCIA RENAL CRÔNICA}

Insuficiência Renal Crônica (IRC) é o estado de disfunção renal persistente, irreversível, geralmente decorrente de um processo patológico, lentamente progressivo. Às vezes, no entanto, o estado crônico de falência renal instala-se rapidamente após uma agressão renal aguda, capaz de deteriorar os rins de forma irreversível, tal como acontece em dois exemplos clássicos - a necrose cortical aguda e glomerulonefrite rapidamente progressiva (ENGEL et al., 2005).

A IRC é uma doença silenciosa, que não apresenta sinais e sintomas prévios significativos. Estes se manifestam e são percebidos quando a patologia está instalada no organismo. A sintomatologia surge de forma inesperada, em fases mais avançadas da doença, submetendo a pessoa a tratamentos que exigem mudanças de hábitos de vida (TOMÉ, 2011).

A IRC é caracterizada pela filtração glomerular menor que $90 \mathrm{~mL} / \mathrm{min} / 1,73 \mathrm{~m}^{2}$, durante um período de três meses ou mais, pela incapacidade dos rins em manterem o equilíbrio metabólico e hidroeletrolítico, resultando em uremia. O tratamento da IRC depende da evolução da doença, que pode ser conservador com o uso de medicamentos, dietas e restrição hídrica, ou com terapias de substituição renal, hemodiálise, diálise peritoneal e transplante renal (GRICIO et al., 2009).

Existem inúmeros exemplos de nefropatias que podem levar à perda progressiva da função renal. Todas elas após um período variável (de 3 a 20 anos, em média), evoluem para um estado conhecido como Doença Renal em Fase Terminal (DRFT), definida pela queda irreversível da função renal a níveis residuais ( $<15 \%$ da função normal). Nesta fase, a histopatologia renal perde as características específicas das fases iniciais da nefropatia, apresentando uma alteração universal: a fibrose glomerular e intersticial, associada à degeneração ou atrofia dos néfrons (FASSINI et al., 2011).

O paciente, então, apresenta os diversos sinais e sintomas que compõem a denominada Síndrome Urêmica Grave, todos decorrentes da perda quase completa da função renal. Neste momento, torna-se 
imprescindível uma terapia de substituição renal, representada pela diálise ou pelo transplante renal (FASSINI et al., 2011).

O número de pacientes com DRFT tem aumentado progressivamente com o passar dos anos em diversos países. No Brasil, a hemodiálise é o tratamento mais utilizado para IRC, numa proporção de $90 \%$ das diálises. A IRC pode acometer qualquer idade, sexo ou raça (SESSO et al., 2007).

A IRC tem como principais complicações o aumento da ureia no sangue (azotemia), a qual desencadeia uma série de sinais e sintomas conhecidos como uremia ou síndrome urêmica. As causas principais podem ser: pré-renal (em decorrência da isquemia renal); renal (consequente de doenças como as glomerulopatias, hipertensão arterial, diabetes etc); pós-renal (em virtude da obstrução do fluxo urinário) (FERMI, 2008).

\section{METODOLOGIA}

Foi realizada busca bibliográfica utilizando as bases de dados da Scientific Electronic Library Online (SCiELO) e da Sociedade Brasileira de Nefrologia, envolvendo os periódicos do período de 2002 até 2015, referentes à literatura existente sobre os tipos de insuficiência renal e o estadiamento da doença aguda à crônica. Os descritores foram: insuficiência renal, estadiamento e terapias para tratamento, sendo a língua portuguesa definida como fonte de pesquisa, excluindo artigos que não tinham relação com o tema ou publicados no período anterior a 2002.

\section{RESULTADOS E DISCUSSÃO}

Foram pesquisados artigos disponibilizados no banco de dados da SCiELO e da Sociedade Brasileira de Nefrologia, envolvendo os temas relacionados aos tipos de insuficiência renal e a sua evolução da insuficiência renal aguda para crônica.

Um novo sistema de estadiamento da IRC proposto pela National Kidney Foundation, considera os seguintes estágios (KDOQI, 2002):

Quadro 1: Estágios da IRC.

\begin{tabular}{|c|c|c|c|}
\hline \multicolumn{2}{|c|}{ Estágio 1} & \multicolumn{2}{|c|}{$\begin{array}{c}\text { TFG ? } 90 \mathrm{~mL} / \mathrm{min} / 1,73 \mathrm{~m}^{2} \\
\text { Lesão renal crônica em insuficiência }\end{array}$} \\
\hline \multicolumn{2}{|c|}{ Estágio 2} & \multicolumn{2}{|c|}{$\begin{array}{c}\mathrm{TFG}=60-89 \mathrm{~mL} / \mathrm{min} / 1,73 \mathrm{~m}^{2} \\
\text { Insuficiência renal crônica LEVE }\end{array}$} \\
\hline \multicolumn{2}{|c|}{ Estágio 3} & \multicolumn{2}{|c|}{$\begin{array}{c}\mathrm{TFG}=30-59 \mathrm{~mL} / \mathrm{min} / 1,73 \mathrm{~m}^{2} \\
\text { Insuficiência renal crônica MODERADA }\end{array}$} \\
\hline Estágio 4 & \multicolumn{2}{|c|}{$\begin{array}{c}\mathrm{TFG}=15-29 \mathrm{~mL} / \mathrm{min} / 1,73 \mathrm{~m}^{2} \\
\text { Insuficiência renal crônica GRAVE }\end{array}$} & \\
\hline Estágio 5 & \multicolumn{2}{|c|}{$\mathrm{TFG}<15 \mathrm{~mL} / \mathrm{min} / 1,73 \mathrm{~m}^{2}$} & \\
\hline
\end{tabular}


Segundo Gomes et al. (2005), pacientes com $\mathrm{ClCr}<30 \mathrm{~mL} / \mathrm{min} / 1,73 \mathrm{~m}^{2}$ apresentaram maior nível de Hormônio Paratiroidiano Intacto (iPTH), apesar de valores normais para cálcio, fósforo, fosfatase alcalina e $\mathrm{tCO}_{2}$. Pacientes com valores de iPTH duas vezes acima do valor superior da normalidade $(144 \mathrm{pg} / \mathrm{mL})$ apresentaram menor valor de $\mathrm{tCO}_{2}$. A fosfatase alcalina óssea foi avaliada em 37 pacientes com $\mathrm{ClCr}<30$ $\mathrm{mL} / \mathrm{min} / 1,73 \mathrm{~m}^{2}$, mostrando correlação com a fosfatase alcalina, mas não com o iPTH. Biópsia óssea em nove pacientes com $\mathrm{ClCr}<30 \mathrm{~mL} / \mathrm{min} / 1,73 \mathrm{~m}^{2}$ e $\mathrm{iPTH}>144 \mathrm{pg} / \mathrm{mL}$ mostrou osteíte fibrosa (4), lesão mínima (4) e alto remodelamento (1).

Para Silva (2011), os resultados mostram que, para aumentar a provisão de acesso vascular arteriovenoso antes do início da hemodiálise no Brasil, os esforços devem ser focados no cuidado pré-diálise.

A IRC condiciona o paciente a realizar terapias de substituição da função renal na forma da diálise peritoneal, hemodiálise ou transplante. Por ser uma doença progressiva e silenciosa, seu diagnóstico, na maioria dos casos, só é feito na fase terminal, requerendo de imediato TRS. A doença em si e o tratamento desencadeiam uma sucessão de situações conflituosas, que compromete o cotidiano do paciente, bem como de seus componentes familiares, impondo-lhes adaptações e mudanças no estilo de vida. Na maioria das vezes, a pessoa numa condição de portador crônico de alguma patologia necessita compartilhar este enfrentamento com sua família ou com outras pessoas próximas, buscando ajuda e apoio, pois esta situação requer readaptação individual e familiar. É importante ressaltar, porém, que as estruturas familiares nem sempre dão conta, sozinhas, de serem sustentáculos destas situações. Elas precisam do apoio dos profissionais de saúde, bem como de suporte e colaboração de outras pessoas da sua comunidade (SILVA et al., 2009).

As alterações na vida dos pacientes são, particularmente, incômodas, contínuas para eles, uma vez que podem se sentir diferentes e excluídos, por serem proibidos de comer certos alimentos, terem uma ingesta hídrica reduzida e controlada, necessitarem de remédios continuamente e serem submetidos ao tratamento dialítico para a manutenção de suas vidas. Nesta perspectiva, torna-se necessário realizar terapêutica contínua, incluindo atividades sócio-educativas com esses pacientes para que eles tenham maior conhecimento sobre a IRC e seu tratamento, adquiram segurança e maiores subsídios para o autocuidado e, assim, tenham melhor adesão ao tratamento (MEIRELES, 2004).

Apesar do estabelecimento de rotinas para controle, as infecções virais e bacterianas continuam sendo a maior causa de morbidade e mortalidade nos pacientes com IRC, principalmente naqueles em TRS. As infecções contribuem com 30 a $36 \%$ das mortes dos pacientes em diálise, entretanto, muitas destas mortes poderiam ser prevenidas por vacinas (RANGEL, 2006).

Segundo Bastos e Kirsztajn (2011), o diagnóstico precoce, encaminhamento imediato e instituição de medidas para diminuir/interromper a progressão da IRC estão entre as estratégias-chave para melhorar os desfechos.

Em suma, quando se trata da evolução desta doença, ou seja, o estadiamento, não se pode esquecer dos 
três pilares de apoio da IRC, que são: 1) diagnóstico precoce da doença, 2) encaminhamento imediato para tratamento nefrológico e, 3) implementação de medidas para preservar a função renal (BASTOS; KIRSZTAJN, 2011).

\section{CONSIDERAÇÕES FINAIS}

No atual contexto evolutivo da insuficiência renal de estágio a estágio, é papel do especialista em nefrologia fazer as devidas orientações, realizar em seu ambiente de trabalho com a comunidade local, clientes e familiares a prevenção de doenças renais. Outras medidas são muito importantes também, como fazer diagnóstico e tratamento de hipertensão arterial, além de realizar o diagnóstico e tratamento de infecções urinárias, dentre outras atribuições.

Faz-se necessário orientar para a prevenção da doença, antes da insuficiência renal aguda atingir o estágio cinco, ou seja, a falência renal crônica. O foco é salvar vidas e diminuir gastos com hospitalização e tratamento, que no país tem um alto custo.

De acordo com a Sociedade Brasileira de Nefrologia, é recomendável a pacientes com mais de 40 anos fazerem anualmente uma consulta médica com um médico nefrologista, ou seja, realizar exames de dosagem da creatinina no sangue e o exame de urina. Também são importantes novas pesquisas e novos estudos neste assunto, tendo em vista alternativas eficazes de tratamento da doença.

\section{REFERÊNCIAS}

BASTOS, Marcus Gomes; KIRSZTAJN, Gianna Mastroianni. DRC: diagnóstico precoce, encaminhamento imediato e abordagem interdisciplinar em pacientes não submetidos à diálise. In: Rev. Bras Nefrol 2011;33(1): 93-108.

BORDEI P,; SAPTE, E.; ILIESCU, D. Double renal arteries originating from the aorta. Surg Radiol Anat 2004; 26(6):474-9.

BRASIL. Ministério da Saúde. Secretaria de Atenção à Saúde. Departamento de Atenção Básica. Prevenção clínica de doenças cardiovasculares, cerebrovasculares e renais. - Brasília: Ministério da Saúde, 2006. 56 p. - (Cadernos de Atenção Básica; 14) (Série A. Normas e Manuais Técnicos).

ENGEL, C. L.; MARINHO, M. L.; DURAND, A; ENGEL, H.; LIMA, M. R. Do Internato à Residência Nefrologia. Volume 5: insuficiência renal aguda, insuficiência renal crônica e Terapia de substituição renal. Medcurso, 2005.

ETZEL MR. Manufacture and use of dairy protein fractions, J. Nutr., v. 134, n. 4, p. 996-10002, 2004.

FASSINI, Aline et al. Doença Renal Crônica: Planejamento e Gerência em Saúde III. Universidade Federal Fluminense. Faculdade de Medicina. Departamento de Saúde da Comunidade, 2011.

FERRAZ, R.R.N; DEUS, R.B. Incidência de insuficiência renal aguda na Unidade de Terapia Intensiva Neonatal de um hospital paulista. In: Acta paul. enferm. vol.22 no.spe1 São Paulo 2009. 
GOMES, C.P. et al. Comprometimento ósseo de pacientes portadores de doença renal crônica em tratamento conservador. São Paulo Med. J. v.123 n. 2 São Paulo mar. 2005.

GODOY, F.G; SILVA, A.M. . Incidência de Clearance de Creatinina com valores reduzidos: Uma ferramenta para o diagnósticos de Insuficiência Renal Crônica. XIII Encontro Latino Americano de Iniciação Científica e IX Encontro Latino Americano de Pós-Graduação Universidade do Vale do Paraíba. São José dos Campos: Univap, 2006.

GRICIO, T. C., KUSUMOTA L.; CÂNDIDO, M. L. Percepções e conhecimentos de pacientes com doença renal crônica em tratamento conservador. Rev. Eletr. Enferm. 2009 [acesso 2011 Set 20]; 11(4):884-93.

K/DOQI. KIDNEY DISEASE OUTCOME QUALITY INITIATIVE. Clinical practice guidelines for chronic kidney disease: evaluation, classification and stratification. Am J Kidney Dis. 2002;39(Suppl 2):S1-S246.

MAGRO, Márcia Cristina da Silva; VATTIMO, Maria de Fátima F. Avaliação da função renal: creatinina e outros biomarcadores. Rev. bras. ter. intensiva v.19 n.2 São Paulo abr./jun. 2007..

MOON, I.; KIM, Y.; PARK, J.; KIM, S.; KOH, Y. Various vascular procedures in kidney transplantation. Transplant Proc 1998; 30(7):3006.

MOTTA, Valter T. Bioquímica. 2a ed. Rio de Janeiro: MEDBOOK, 2011.

NOLAN, C. R., ANDERSON, R. J. Hospital-acquired acute renal failure. J Am Soc Nephrol, 2005;9:710-718.

NUNES, G.L.S. Avaliação da função renal em pacientes hipertensos. Rev Bras Hipertens vol.14(3): 162-166, 2007.

OZKAN, U.; O'UZKURT, L; TERCAN, F.; KIZILKILIÇ, O.; KOÇ, Z.; KOCA, N. Renal artery origins and variations: angiographic evaluation of 855 consecutive patients. Diagn Interv Radiol 2006; 12(4):183-6.

SESSO, R. et al. Resultados do censo de diálise da SBN, 2007. J Bras Nefrol 2007; 29:197-202.

SILVA, Gisele Macedo da. Acesso vascular permanente em pacientes renais crônicos terminais no Brasil. Rev. Saúde Pública vol. 45 nº.2 São Paulo abr. 2011 Epub 11-Fev-2007 e 2011.

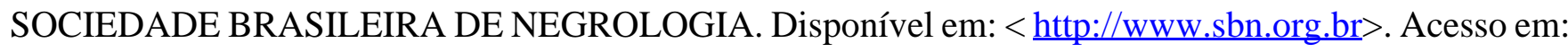
20 dez. 2013.

STEVENS, L. A.; LEVEY, A. S. Measurement of kidney function. Med Clin Am 2005;80:457-73.

TESTUT, L.; LATARJET, A. Cavidad abdominal y su contenido, 1978. In: PALMIERI, Breno José et al. Rev. Col. Bras. Cir. vol.38 no.2 Rio de Janeiro Mar./Apr. 2011. 
${ }^{[1]}$ Enfermeira graduada pela UNIDERPE, concluinte do curso de Especialização em Nefrologia.

${ }^{[2]}$ Biólogo. Doutor em Teoria e Pesquisa do Comportamento. Docente e Pesquisador do Instituto Federal do Amapá - IFAP.

[3] Biomédica. Doutora em Doenças Tropicais. Docente e Pesquisadora da Universidade Federal do Amapá, AP. Pesquisador colaboradora do Núcleo de Medicina Tropical da UFPA (NMT-UFPA).

${ }^{[4]}$ Doutora em Psicanálise Clínica, Pesquisadora pelo Centro de Pesquisa e Estudos Avançados.

${ }^{[5]}$ Bióloga. Doutora em Ciências Biológicas - Área de Concentração Genética. Professora e Pesquisadora no CESUPA - Centro Universitário do Estado do Pará.

[6] Biólogo. Doutor em Medicina/Doenças Tropicais. Docente e Pesquisador na Universidade Federal do Pará - UFPA. Pesquisador no Laboratório de Toxicologia Humana e Ambiental e no Laboratório de Estresse Oxidativo do Núcleo de Medicina Tropical da UFPA (NMT-UFPA).

\section{PUBLIQUE SEU ARTIGO CIENTÍFICO EM:}

https://www.nucleodoconhecimento.com.br/enviar-artigo-cientifico-para-submissao 